\section{START: Stimulus and response tools for experiments in memory, learning, cognition, and perception}

\section{STEPHEN A. POFFEL and ROBERT J. GREGORY University of Idaho, Moscow, Idaho}

This article discusses the main features of a computerized instructional package for experimental psychology applications.

\section{Goals of the Package}

One goal common to many psychology departments is to provide undergraduate students with "hands-on" laboratory experience. This experience normally includes the design, execution, and reporting of one or more psychological experiments. One major dilemma, faced by nearly all instructors, is the creation of a feasible and interesting research application that can be accomplished in a limited amount of laboratory time. This dilemma is amplified by the need for specialized research equipment and high-level skills to operate that equipment.

To overcome some of these problems, we developed START: Stimulus and Response Tools for Experiments in Memory, Learning, Cognition, and Perception. The package consists of 11 computer programs written in Applesoft BASIC for the Apple II and Apple Ile series microcomputers. Although a printer is optional, $48 \mathrm{~K}$ of memory is required. One program requires a color monitor.

The programs and manual facilitate the collection of meaningful data in a short amount of time with small sample sizes $(\mathrm{N} \leq 10)$. To create a limited form of problem solving, we have deliberately provided unstructured projects. The specific hypotheses and actual research design (independent and dependent variables, etc.) are left to the instructor and students. These issues usually depend upon subject and time resources. Students may use themselves or fellow laboratory students as subjects.

\section{A Brief Annotated List of Programs}

Reaction Time. This program provides simple stimuli for testing three types of reaction time (RT): simple, choice, and identification. Program variables are the type of RT, number of trials, type of stimuli, and the delay interval between the ready signal and the stimulus.

Precognition. This program is a study in parapsychology. After the subject enters a guess, an integer

This work was funded largely by NSF Grant SER 8161015. The authors' mailing addresses are: Stephen A. Poffel, Department of Psychology, Washington State University, Pullman, WA 99164; Robert J. Gregory, Department of Psychology, University of Idaho, Moscow, ID 83843. between 1 and 5 is randomly generated. The subject is allowed one prediction for each trial, and total performance is recorded.

Prototype Formation. This program studies the prototype formation theory of memory. Simple line shapes are presented on the screen. Later, the subject must recall which shapes were presented. At the end, a graph pictorially represents the subject's responses.

Problem Solving. The hobbits and orcs (cannibals and missionaries) problem is graphically presented. The subject's task is to guide six characters across a river following predefined rules. The program tests each move for validity and records moves, decision times, and errors. These are output at the end of the program.

Pitch Memory. The computer can be used as is or with a simple modification to provide for an external speaker. One or more subjects simply mark an answer sheet as they are tested for memory of musical tones.

Poggendorff. A good program for the topic of visual illusions. The subject's score is tabulated as the left diagonal of the illusion is adjusted to match a direct continuation of the right diagonal. Program variables are the number of trials, type of Poggendorff illusion (e.g., wide, narrow, acute, obtuse), and the length of diagonals.

T-Scope. A tachistoscopic simulation in which words are presented for brief durations. The visual duration threshold is displayed following each correct response. Specialized words may be selected by the experimenter and inserted into the program.

Dot-Enumeration. The subject monitors presentations of an asterisk and enters perceived locations on a grid. The program computes the degree of error between presented and perceived locations and, at the end, displays an error map for left and right visual fields.

Scaling. The subject constructs a pitch scale using the equal-appearing method of intervals. Program variables are the upper and lower tones. Results include high and low tones with intervening quartiles.

Probability Learning. A naive subject demonstrates probability learning by attempting to predict on which side of the screen a stimulus will appear. The experimenter controls the percentage of presentations to the left and right side. Program variables are the number of trials and percentage of left and right guesses.

Same Different. The subject's task is to distinguish between pairs of stimuli and respond same or different. Program variables are the number of trials, interstimulus interval, and the type of stimuli: shape, color, or pitch.

\section{Laboratory Manual}

A laboratory manual accompanies each program package. The manual is written for both the instructor and the student. Basically, one chapter is devoted to each concept (i.e., Reaction Time, T-Scope, etc). Each chapter provides the following information: 
Overview-provides topic background and a brief literature review for each program.

Program operation-explains the programmed options. For example, the type of RT or the configuration of the Poggendorf illusion can be selected by the studentexperimenter. Examples of input and output are provided.

Research projects-provides general guidelines for several possible projects and usually includes the "classic" from which the program was modeled.

Readings-each chapter contains a separate, briefly annotated, reference section related to the suggested research projects.

\section{Sample Classroom Application: \\ Program Reaction Time}

To use the Reaction Time module, the instructor begins by giving a background lecture on the use of RT in psychology, borrowing from the overview section of the manual and related materials. The manual discusses the history of RT from Donders to the present, illustrates the subtraction paradigm for partitioning $\mathrm{RT}$, and clarifies basic concepts such as simple versus choice RT.

The laboratory class is then subdivided into groups of two to four students. The students are given the purposefully vague assignment of designing a useful project using RT as some sort of a dependent variable. They are referred to the "Research Projects" section of the assigned chapter, but expressly told that these are only guidelines. In fact, most instructors encourage students to invent novel projects. Nonetheless, inexperienced or unimaginative students can always fall back on one of the three research projects given in the manual: correlation of RT with intelligence, daily rhythms in RT, or the functional relationship between RT and the number of choice alternatives.

The next step is tutorial interaction between student groups and instructor. Using the outline for a research proposal provided in the manual, the instructor discusses the research proposal with each student group, providing hints on where unforeseen problems might occur and asking questions to clarify vague points. The research proposal outline includes the following items: title of project, central hypothesis, type of design, statistics, indepen- dent and dependent variables, control variables, and anticipated problems. The students then collect their data and write individual research reports with the aid of an outline provided in the manual.

The actual data collection is a very straightforward process of interactively entering the program desired, the number of trials, the type of RT, the type of stimuli, and the delay interval, and then obtaining keypress RTs to simple visual stimuli from one subject at a time.

The diskette provided with START automatically presents a menu when the system is turned on. After selecting the Reaction Time program, the studentexperimenter is presented an extensive instructions screen. This screen can be accessed at any point prior to the seating of the subject, and the various parameters (e.g., choice of delay interval, number of trials) can easily be altered or corrected. Erroneous experimenter inputs (e.g., entering the letter " $O$ ' instead of the number zero) are detected, and the user is prompted to try again. The program has been "crash-proofed" against all the different aberrant inputs that we could imagine.

After the student has entered the desired parameters for the experiment, the instructions screen for the subject appears and the first subject may be seated. Depending upon the type of RT being investigated, the subject is instructed to press the space bar whenever a certain stimulus appears (simple RT), press the space bar when one stimulus appears but do nothing for other stimuli (identification RT), or press the corresponding key when one of the digits 1-8 appears (choice RT).

This program records the RTs to the nearest $.01 \mathrm{sec}$ and later asks the experimenter if printer or screen output is desired. Finally, the program asks if more subjects are to be tested. If the answer is "yes," the program starts over; otherwise control is returned to the menu.

\section{Availability}

START is available from CONDUIT, Iowa City, Iowa 52242. The price of the laboratory manual and software on diskette is $\$ 150.00$; additional manuals are $\$ 7.00$ each, and additional software copies are $\$ 10.00$ each.

(Revision accepted for publication August 17, 1984.) 